\title{
Erwin Rauscher
}

Pädagogische Hochschule Niederösterreich, Campus Baden

\section{Vom Kopf auf die Füße!?}

\section{Eine Prophetin in der Prophetiefalle}

DOI: https://doi.org/10.53349/sv.2021.i3.a143

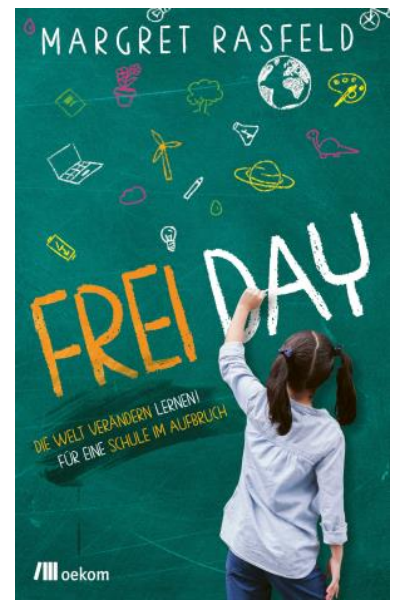

Margret Rasfeld

\section{FREI DAY}

Die Welt verändern lernen!

Für eine Schule im Aufbruch

oekom verlag, München 2021

ISBN 978-3-96238-294-0

Margret Rasfeld, inzwischen in Pension befindliche Schulleiterin aus Berlin, als Schulinnovatorin weit über die Grenzen hinaus bekannt und berühmt geworden, nutzt den Zeitgeist einer viel jüngeren Revolutionärin aus Schweden, um mit einem Wortspiel aus Friday for Future den FREI DAY für Schule zu proklamieren und rhetorisch brilliant ein Schulleben zu inszenieren, das Bildung neu denken und neu lenken, ja sogar die Welt verändern will - zukunftskompetent und ohne Leistungsdruck in der Wissensvermittlung. Ein lesenswertes und doch nachdenklich machendes Buch für eine Schöne Neue Welt der Schule. 


\section{Die Zukunft wartet nicht!}

Welche ein Aufbruch der schönen Worte, Kraft spendend, Mut machend: „Das Wichtigste [...] sind [...] die Kinder und Jugendlichen [...], gleichwertige, gleichwürdige, aber unterschiedliche Menschen“ (S. 35). Für sie wird aus dem Berliner Plattenbau ein „Haus des Lernens", in dessen „Lernbüros" die Schüler*innen „selbst entscheiden (können), was sie lernen wollen“ und „losziehen, um das Mutigsein zu lernen“ (S. 45.), dabei „ihre eigenen Erwartungen und Vorstellungen loszulassen und Vertrauen in ergebnisoffene Prozesse zu fassen“ (S. 49).

Der FREI DAY wird zum „Befreiungstag“, dessen Rahmen die SDGs bilden, an dem die Lehrer*innen zu Lernbegleiter*innen werden, an dem die Schüler*innen erfahren, „dass ihr Wissen und Tun Wirkung entfaltet“ (S. 99), an dem „das Leben die Fragen“ (S. 102) stellt, ein Tag der pädagogischen Salutogenese, der "Schulen in nachhaltige Vorzeigeorte verwandeln" (S. 112) und Kinder wie Jugendliche „Inklusion leben und dadurch wachsen“ (S. 120) lassen kann.

Die Be-Geist-erung der Autorin wirkt ansteckend, sie führt „Von digitaler Bildung zu digitaler Mündigkeit“ (S. 133), „Vom Musterbruch zur Normalität" (S. 175). Die zum Buch parallele Website www.frei-day.org berichtet von aktuell (im Dez. 2021) bereits 80 FREI DAY-Schulen und 7835 darin bereits „erreichten“ Schüler*innen. Längst ist banalem Lernen „Schritt für Schritt gemeinsam“ ein „Lernen, die Welt zu verändern“. Ein Mutmachbuch also für alle pädagogisch Begeisterbaren, um die Bildung vom Kopf auf die Füße zu stellen!

\section{Alles Gute ist neu, alles Neue ist gut?}

Seien wir alle der Autorin und Vorbildern wie ihr besonders dankbar, wenn sie uns Visionen vor Augen stellen, die uns helfen, Wirklichkeit zu gestalten, Bildungsgelingensverantwortung zu übernehmen, Zukunftsfähigheit alltagstauglich und gegenwärtig zu machen. Aber muss dafür, wie in diesem beispielstrotzenden und optimistischen Buch, alle Gegenwart der Schule und der Schulen diskreditiert werden? Ist tatsächlich an allen unseren Schulen die Ausgangsfrage zu stellen, „Warum es so nicht weitergehen kann“ (S. 15)? Braucht es dafür eine ganz andere, eine neue Schule? "Deutschland fehlt die Vison“ (S. 59)? Trifft die Frage „Warum ketten wir uns an das alte System?" (S. 57) tatsächlich auch für alle Schulen in der Schweiz und in Österreich zu - jene 80 besonderen ausgenommen? Braucht es das Verdammen des Täglichen und der Vielen, um „neu“ und „anders“ zu denken? Ist es nicht vielmehr eine Falle der Prophetie, wenn innerhalb derselben ein unlösbarer Widerspruch aufgebaut wird zwischen Übernommenem und neu zu Entwickelndem, frei nach dem Motto, nur das Neue daran sei gut, nur das Gute daran sei neu? Entsteht nicht gerade in diesem Gegenüber und Entweder-Oder der Widerstand und Widerspruch, wonach die ständigen Neuerungen nur die Kontinuität und den Konsens ruinieren? Es sei dem*der aufmerksamen Lesenden überlassen, selbst zu urteilen. Dafür sei (durchaus ein wenig hintergründig auf die Veränderung des Distichons „Auf mehrere Bücher. Nach Lessing“ von Johann Heinrich Voß (1751-1826) in seinem Musenalmanach von 1792 verwiesen: „Dein redseliges Buch lehrt mancherlei Neues und Wahres, | Wäre das Wahre nur neu, wäre das Neue nur wahr!" Das Distichon bezieht sich auf 
Lessings „Briefe, die neueste Literatur betreffend“. Im 111. Brief vom 12. 6.1760 heißt es: „Wenn es erlaubt ist, allen Worten einen andern Verstand zu geben, als sie in der üblichen Sprache der Weltweisen haben, so kann man leicht etwas Neues vorbringen. Nur muss man mir auch erlauben, dieses Neue nicht immer für wahr zu halten."

\section{Variatio delectat}

Geschätzte Frau Kollegin Rasfeld! Ihr Tun und Ihre Schule gehören vor jeden Vorhang. Sie stimulieren ideenreich, überwinden Widerstand und Passivität, modellieren Zukunft. Aber Sie tun das nicht alleine - viele Schulen und viele Schulleiter*innen haben sich auf den Weg gemacht: Auch UNESCO-Schulen, Ökolog-Schulen, IMST-Schulen und viele andere mehr, die keine eigenen Namen tragen. Und (fast) alle ihre Lehrer*innen sind besser als ihr Ruf - wenn man sie lässt. Bleiben Sie auch in der Schulentwicklung eine inklusiv Denkende und Handelnde. Aufbruch braucht Durchbruch, nicht Umbruch. Aufbrechen braucht kein Abbrechen, vielmehr Aufzeigen und Aufbauen.

\section{Autor}

Erwin Rauscher, Univ.-Prof. HR MMag. DDr., seit 2006 (Gründungs)Rektor der Pädagogischen Hochschule Niederösterreich; davor seit 1989 Direktor an Gymnasien; Lehraufträge und Gastprofessuren an den Universitäten Klagenfurt, Graz, Salzburg, Linz; Mitglied der Europäischen Akademie der Wissenschaften; Lehrerfortbildner inter/national; Bücher und Zeitschriftenpublikationen zu Schulinnovation und Schulmanagement.

Kontakt: erwin.rauscher@ph-noe.ac.at 